\title{
IMPACT OF STELLAR EVOLUTION ON PLANETARY HABITABILITY
}

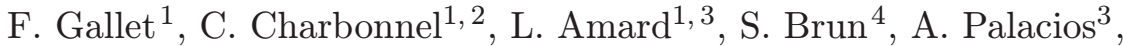 \\ S. Mathis ${ }^{4}$ and E. Bolmont ${ }^{4}$
}

\begin{abstract}
With the ever growing number of detected and confirmed exoplanets, the probability to find a planet that looks like the Earth increases continuously. While it is clear that being in the habitable zone does not imply being habitable, a systematic study of the evolution of the habitable zone is required to account for its dependence upon stellar parameters. In this study, we aim to provide the community with the dependence of the habitable zone upon the stellar mass, age, metallicity, and rotation. We use stellar evolution models computed with the STAREVOL code to study the evolution of the habitable zone. Mass and metallicity are the stellar parameters that have the most dramatic effects on the habitable zone limits. The evolution of the habitable zone limits is also linked to the evolution of the stellar activity (through the Rossby number) that depends on the considered stellar mass and rotation. Using observed trends of stellar magnetic field strength we also constrain the planetary magnetic field (at the zero order) required for a sufficient magnetospheric protection during the whole stellar evolution. We explicit for the first time the systematic dependence of planet habitability on stellar metallicity and rotation along the full evolution of low- and intermediate-mass stars. These results can be used as physical inputs for a first order estimation of exoplanetary habitability.
\end{abstract}

\footnotetext{
${ }^{1}$ Department of Astronomy, University of Geneva, Chemin des Maillettes 51, 1290 Versoix, Switzerland

${ }^{2}$ IRAP, UMR 5277, CNRS and Université de Toulouse, 14, Av. E. Belin, 31400 Toulouse, France

${ }^{3}$ LUPM, UMR 5299, Université Montpellier/CNRS, 34095 Montpellier, France

${ }^{4}$ Laboratoire AIM Paris-Saclay, CEA/DRF - CNRS - Univ. Paris Diderot - IRFU/SAp, Centre de Saclay, 91191 Gif-sur-Yvette Cedex, France
} 


\section{Introduction}

Thanks to the increase in precision of modern observational techniques (e.g. radial velocity and transit method) the number of confirmed exoplanets is now above 2900 (exoplanet.eu and exoplanets.org) and this amount could be increased by including the unconfirmed objects. Among these confirmed exoplanets, about 100 of them are inside of the so called habitable zone (hereafter HZ) of their host star (hzgallery.org) and this number will dramatically rise in the future thanks to Kepler (Borucki et al. 2010), K2 (Howell et al. 2014), and the future PLATO (Rauer et al. 2014), TESS (Ricker et al. 2015), and CHEOPS (Broeg et al. 2013) missions.

Planetary habitability is a complex notion. Many studies consider that surface liquid water is one of the key ingredients to characterize a planet as habitable (Rasool \& de Bergh 1970; Kasting et al. 1988). The presence of surface liquid water depends on many different parameters and the most commonly used one is the orbital distance: whether the planet is in the $\mathrm{HZ}$ or not is generally consider to give an insight on its potential habitability. But, of course, being in the $\mathrm{HZ}$ is not enough. The planetary environment is also determining. For instance, the presence of a protective planetary magnetosphere is thought to have a strong impact in helping life complexification through magnetic shielding (Vidotto et al. 2013; Strugarek et al. 2014, 2015; Vidotto et al. 2015).

The aim of the present work is to study the impact of stellar parameters such as metallicity, mass, age, and rotation all along the evolution of low- and intermediate-mass stars on the limits of the HZ (see Gallet et al. 2017). Such investigations have already been performed by Valle et al. (2014), Truitt et al. (2015) and more recently by Gallet et al. (2016), but in the case of non-rotating stellar models. Here we investigate for the first time the impact of stellar rotation on the evolution of the HZ limits.

\section{Habitable zone and stellar model}

The HZ is generally defined as the region around a star within which surface liquid water can be found on telluric exoplanets. It is defined by Kasting et al. (1993) as

$$
d=\left(\frac{L_{*} / L_{\odot}}{S_{\mathrm{eff}}}\right)^{0.5} \mathrm{AU}
$$

where $L_{*}$ and $L_{\odot}$ are the luminosity of the star and the Sun, respectively, and $S_{\text {eff }}$ is a constant equal to the normalized solar flux required to maintain a given planetary surface temperature. This constant depends on the nature of the planetary atmosphere and whether we consider the inner or outer edge of the HZ. Indeed, the inner edge of the $\mathrm{HZ}$ corresponds to a fully saturated water vapour atmosphere (that corresponds to the runaway greenhouse process, e.g. Kasting et al. 1993) while the outer edge corresponds to an atmosphere with a high $\mathrm{CO}_{2}$ partial pressure (maximum greenhouse). The expression of $S_{\text {eff }}$ was recently 
updated in Kopparapu et al. (2014):

$$
S_{\mathrm{eff}}=S_{\mathrm{eff}, \odot}+a T_{*}+b T_{*}^{2}+c T_{*}^{3}+\cdots,
$$

where $T_{*}=T_{\text {eff }}-5780 \mathrm{~K}$ is a function of the stellar effective temperature $T_{\text {eff }}$.

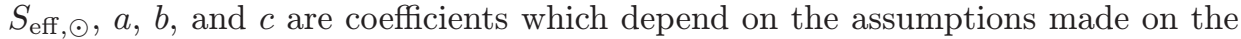
nature of the planetary atmosphere (runaway greenhouse or recent Venus for the inner edge, maximum greenhouse or early Mars for the outer edge).

To study the evolution of the HZ all along the evolution of a planet's host star, we used the stellar structure evolution code STAREVOL to follow the evolution of the main stellar quantities such as radius, effective temperature, and surface angular velocity. We refer to Amard et al. (2016) for a detailed description of the stellar evolution code STAREVOL and of the micro physics inputs (equation of state, nuclear reaction, opacities) as well as the mechanisms that impact the internal transport of chemicals and angular momentum (e.g. Zahn 1992; Maeder \& Zahn 1998; Mathis \& Zahn 2004). We recall here the main assumptions and physical processes included in our model:

- The evolution of angular momentum in the stellar interior is calculated from the first iteration step on the PMS phase and up to the RGB following the formalism developed by Zahn (1992); Maeder \& Zahn (1998); Mathis \& Zahn (2004). This formalism takes into account advection by meridional circulation and diffusion by shear turbulence (see Palacios et al. 2003, 2006; Decressin et al. 2009).

- The convective region is assumed to be in solid-body rotation and magnetic braking is applied following Matt et al. (2015). The mass loss rate is estimated using the prescription of Cranmer \& Saar (2011).

- Star-disc interaction is modelled by a constant surface rotation rate during the early PMS (see Gallet \& Bouvier 2015).

We focus on non-rotating stars with masses ranging between 0.2 and $2 M_{\odot}$ and rotating stars for $0.3,1.0,1.4$, and $2 M_{\odot}$. Figure 1 shows the evolutionary tracks of the non-rotating stars in the Hertzsprung-Russell diagram (hereafter HRD).

\section{Strong dependence of the $\mathrm{HZ}$ limits on the stellar parameters}

In this section we highlight the main results obtained by coupling STAREVOL to the HZ prescription of Kopparapu et al. (2014).

\subsection{Impact of mass and metallicity}

It is well known that the stellar mass, which impacts the stellar luminosity, controls the location of the HZ limits (hereafter HZLs). At a given evolution state, the stellar luminosity and effective temperature increase when the initial mass of the star increases, which moves the HZLs outwards (Eq. (2.1)). In Gallet et al. (2017) 


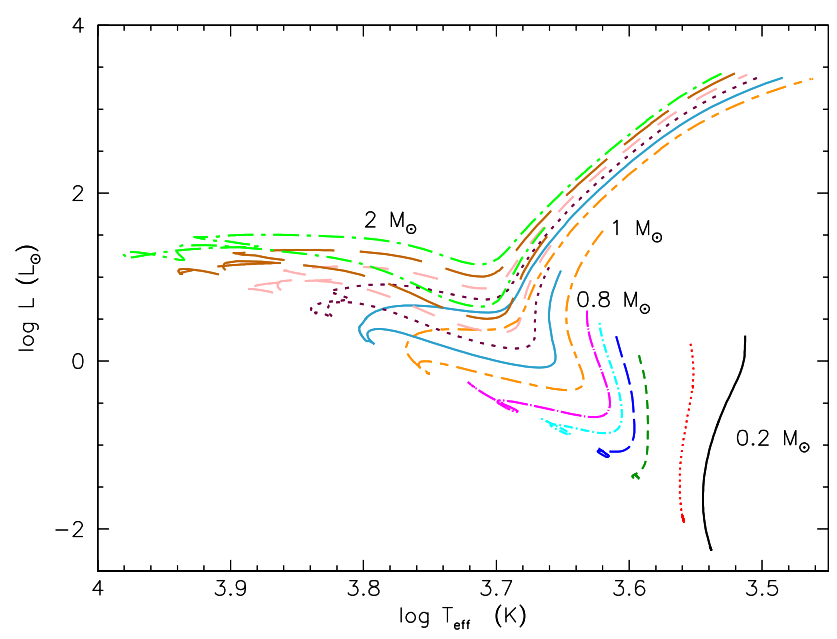

Fig. 1. HRD of non-rotating stars between 0.2 and $2 M_{\odot}$ at solar metallicity. Credit Gallet et al. (2017).

we pointed out that an initial change, even as small as $0.1 M_{\odot}$, in the mass of the star strongly impacts the location of the HZLs.

Moreover we showed, in Gallet et al. (2017), that a change in metallicity has the same effect on the HZLs as a change in initial stellar mass. The main effect of diminishing the initial metal content in the star is to produce a shift, towards higher values, in both luminosity and effective temperature. Indeed, when the metallicity of a star decreases, its overall opacity diminishes as well, due to the reduction of the quantity of metallic bound-free absorption inside the star, which allows an easier escape of energy that leads to the increase of $L_{*}$ and $T_{\text {eff }}$. Figure 2 shows the evolution of the HZLs for different stellar metallicities Z.

In summary, mass and metallicity are thus two stellar parameters that strongly control the evolution of the HZLs.

\subsection{Rotation, stellar activity, and magnetic protection}

In addition to the non-rotating models we took into account the evolution of the rotation in the STAREVOL model for the $0.3,1.0,1.4$, and $2.0 M_{\odot}$ cases.

As the mass and effective temperature, the angular velocity is an intrinsic stellar quantity. In the framework of the conservation of angular momentum, the angular velocity follows the evolution of the stellar structure. It increases when the star is contracting (pre-main sequence hereafter PMS) and reduces when the star expand (red giant branch hereafter RGB). Additionally there are physical mechanisms, such as the stellar winds (Matt et al. 2015), that move the star from such an angular momentum conservation which complicate the evolution of the angular velocity and internal transport processes like meridional circulation and shear mixing (Gallet \& Bouvier 2013, 2015; Amard et al. 2016). The main effect 


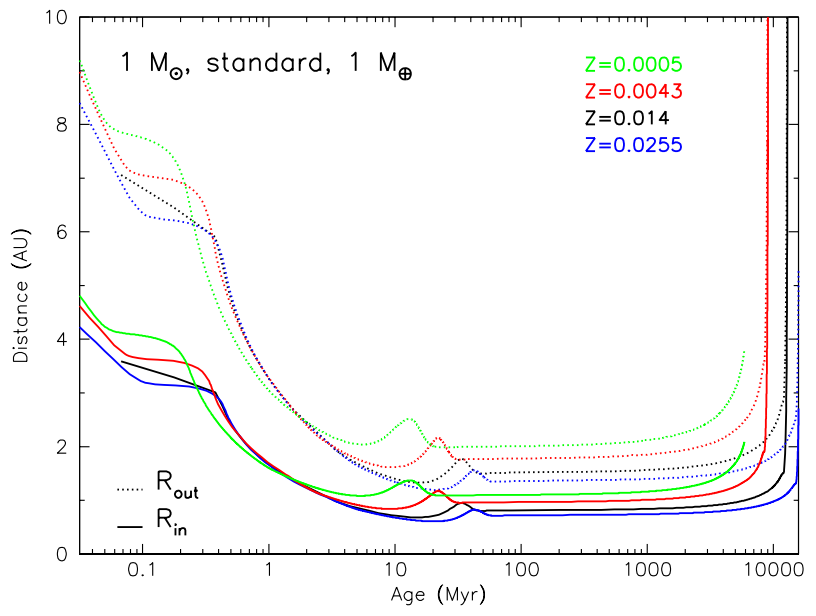

Fig. 2. Effect of the metallicity on the evolution of the HZLs of a $1 M_{\oplus}$ planet orbiting a $1 M_{\odot}$ star. Credit Gallet et al. (2017).

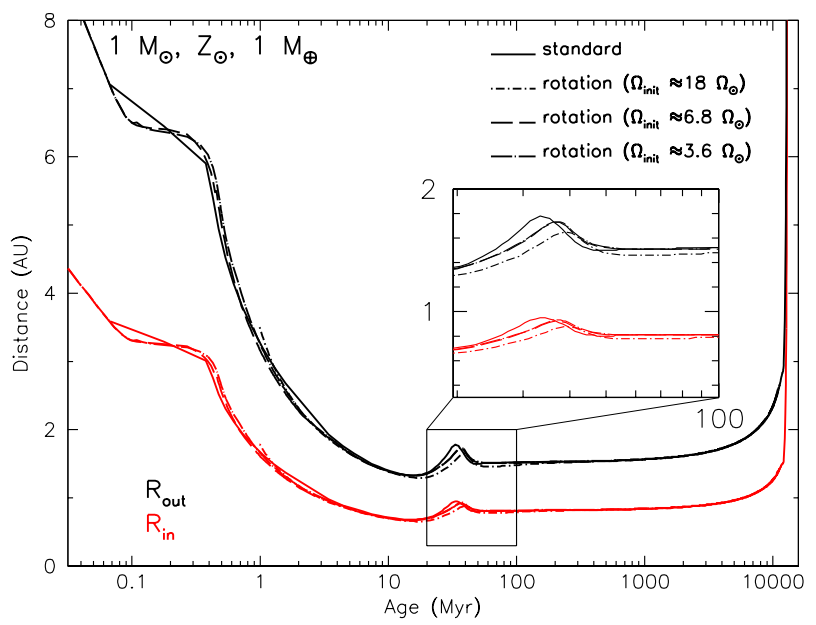

Fig. 3. Effect of the rotation on the evolution of the HZLs in the case of a $1 M_{\oplus}$ orbiting a $1 M_{\odot}$ star with solar metallicity. Credit Gallet et al. (2017).

of a rise of rotation rate is to globally decrease, at a given evolution state, the effective temperature due to centrifugal effects that tend to increase the stellar equatorial radius.

Figure 3 shows that initial stellar rotation has only a marginal effect on the evolution of the HZ around a solar-type star. This figure displays the evolution of $R_{\text {in }}$ and $R_{\text {out }}$ in the case of a 1 Earth mass planet orbiting a $1 M_{\odot}$ star at solar metallicity. We compare four cases: a non-rotating star, a slow $\left(\Omega_{\text {init }}=3.6 \Omega_{\odot}\right)$, a 

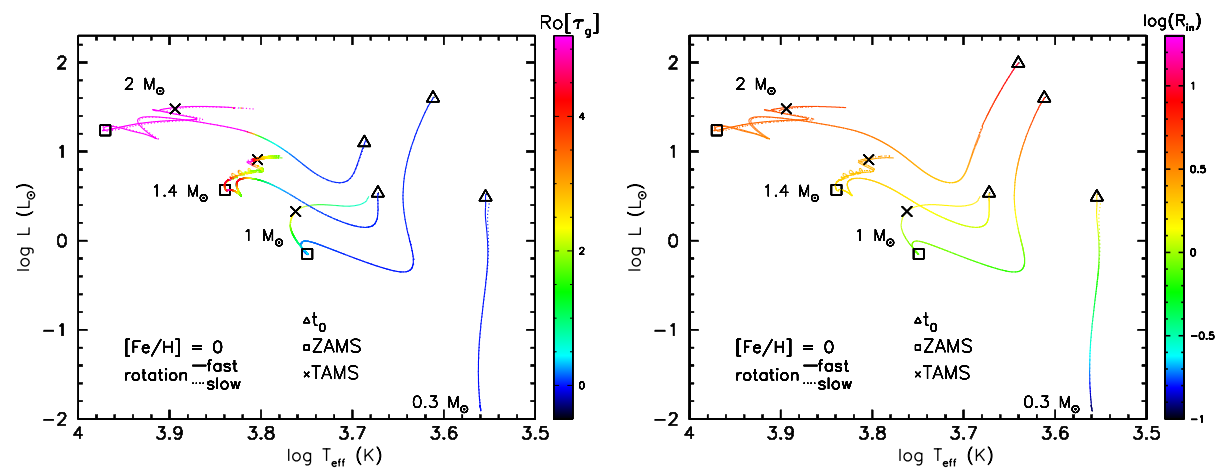

Fig. 4. Variation of the global Rossby number (colour-coded) (left) and $R_{\text {in }}$ (colourcoded) (right) along the evolutionary tracks in the HRD for the $0.3,1,1.4$, and $2 M_{\odot}$, $Z_{\odot}$ models for the fast and slow rotating cases (solid and dashed lines respectively). The triangles, squares and crosses represent the location in the HRD of the age of the first time-step $t_{0}$ in each STAREVOL models, the ZAMS and end of the main-sequence (hereafter MS), respectively. Credit Gallet et al. (2017).

median $\left(\Omega_{\text {init }}=6.8 \Omega_{\odot}\right)$, and a fast $\left(\Omega_{\text {init }}=18 \Omega_{\odot}\right)$ rotating stars. The maximum effect of rotation is seen at the zero-age main sequence (hereafter ZAMS), i.e. around $50 \mathrm{Myr}$ in the case of a $1 M_{\odot}$ star (see the insert of Fig. 3). It coincides with the moment when the stellar contraction stops, which corresponds to the moment when the surface rotation of the star reaches its maximum value. While this effect is quite small, we still can note that HZLs tend to move outward for a decreasing initial angular velocity.

However one of the by-products of rotation is the stellar dynamo, which is linked to the stellar activity. One way to indirectly estimate the stellar activity is to estimate the Rossby number

$$
R \mathrm{o}=\frac{P_{\mathrm{rot}}}{\tau_{\mathrm{conv}}}
$$

with $P_{\text {rot }}$ the surface rotation period of the star and $\tau_{\text {conv }}$ the convective turnover timescale. In this work we used the global turnover timescale within the stellar convective envelope and the angular velocity of the star self-consistently given by STAREVOL. Rossby number lower than unity trace star in strong stellar dynamo regime (strong activity) while Rossby greater than one trace weak dynamo regime. Figure 4 (left) shows the evolution of $R$ o along the stellar evolution of a $0.3,1.0$, 1.4, and $2.0 M_{\odot}$ stars and Figure 4 (right) shows the corresponding evolution of the inner edge of the HZ the outer edge globally follows the same evolution, see Gallet et al. (2017). The main result of this analysis is that stars appear to be in a strong stellar dynamo (stellar activity) regime (Fig. 4, left) during the PMS (first few Myrs of the stellar life) during which the HZLs are the closest to the star (Fig. 4, right). This raises the question of the magnetic protection of the planet itself. 


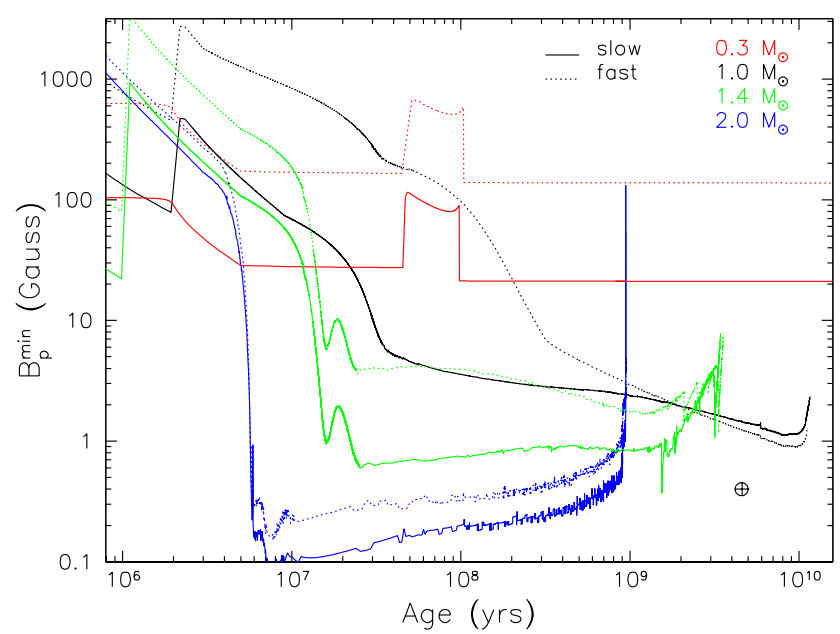

Fig. 5. Minimum planetary magnetic field required by a $1 M_{\oplus}$ orbiting a $0.3,1,1.4$, and $2 M_{\odot}$ at $1 \mathrm{AU}$ to produce an effective magnetic protection. Solid and dashed lines represent slow and fast rotators, respectively. The Earth is represented by the $\oplus$ symbol. Credit Gallet et al. (2017).

The minimum planetary magnetic field required for a strong enough magnetic protection is given by Vidotto et al. (2013) as

$$
B_{p}^{\min } \propto \frac{B_{*} R_{*}^{2}}{R_{\text {orb }}^{2}},
$$

where $B_{*}$ and $R_{*}$ are the stellar magnetic field strength and stellar radius, and $R_{\text {orb }}$ the orbital radius of the planet. Vidotto et al. (2013) used the reconstructed surface magnetic field maps of active M-dwarf (Donati et al. 2006) to determine the magnetic pressure at the planet orbit in order to estimate the largest size of the planetary magnetosphere and thus its magnetic field. We used the observed trend of Folsom et al. (2016) that links the stellar magnetic field strength to the Rossby number to constrain the temporal evolution of the minimum magnetic field $B_{p}^{\text {min }}$ in the case of a $1 M_{\oplus}$ orbiting a $0.3,1.0,1.4$, and $2.0 M_{\odot}$ stars (see Fig. 5).

Figure 5 shows that during the PMS the planetary magnetic field has to be several orders of magnitude higher than the current magnetic field of the Earth $(\approx 0.5 \mathrm{G})$ to efficiently protect the atmosphere of the planet. The magnetic field also has to be stronger if the planet orbits a fast rotator than if it orbits a slow rotator (because of a stronger dynamo in fast rotators as $R o \propto \Omega_{*}^{-1}$ ). While the required magnetic field of explanets around fully convective stars is the strongest, for exoplanets around partly convective star (i.e. stars with mass above $\approx 0.3 M_{\odot}$ ) this minimum magnetic field increases for a decreasing stellar mass. This effect is mainly due to the decrease of the Rossby number, and thus indirectly to the increase of the magnetic activity, towards partly convective lower mass stars. It is 
worth noting that in Figure 5 the planet is fixed at a distance of $1 \mathrm{AU}$. However in some configurations the planet can migrate inward or outward for instance because of the tidal interaction between the planet and the star (Bolmont \& Mathis 2016). Indeed, when $R_{\text {orb }}$ decreases, the planetary magnetic field has to be higher to protect the atmosphere of the planet as shown by Equation (3.2). It is worth noting that a distinction between intrinsic and induced magnetosphere must be done (Russell 2003). Indeed, even without any dynamo process the ionosphere of the planet could play the role of a shield. The efficiency of this shield depends on the direction of the $B_{z}$ of the stellar wind (because of the possible magnetic reconnection, see Russell 2003, for a more detailed discussion).

\section{Dissipation of tidal waves in stellar interiors}

Detailed description of the physics behind the tidal interaction of star with planets can be found in Bolmont \& Mathis (2016). Tidal dissipation, with other interactions, which drives the secular orbital and rotational evolution in star-planet systems takes place inside both star and planet. Its understanding and modelling requires detailed knowledge of the internal structure of both objects. We only consider here the tidal dissipation occurring in the convective envelope of the star by using the formalism of Ogilvie (2013) and Mathis (2015b) that expresses the frequency-averaged dissipation as a function of the size and mass of the radiative core, and the rotation of the envelope, which is here assumed to be uniform. We analyse for the first time the evolution of this dissipation by taking into account simultaneously the structural and rotational evolution of the star.

Figure 6 shows the evolution of the modified quality factor as a function of time in the case of a rotating $1 M_{\odot}$ star. The modified tidal quality factor $Q^{\prime}=3 /(2 \times \mathcal{D}$ (Mathis 2015a) is the inverse of the tidal dissipation $\mathcal{D}$, which gives the response of an object to the tidal potential created by a perturber (Mathis 2015a). The black line represents the evolution of the normalised quality factor where only the structural evolution of the star (size and mass of the radiative core, and the total radius) is included while for the red line both structure and rotation are taken into account. Figure 6 shows that stellar rotation has a dramatic effect on the evolution of the tidal dissipation. During the pre-main sequence the modified quality factor is controlled by the evolution of the internal structure (see the similarity of the red and black curves in Fig. 6) while during the MS its evolution is mainly controlled by the rotation. Indeed, during this stellar phase, the internal structure remains almost constant while the surface of the star is braked by the stellar winds (Gallet \& Bouvier 2015; Amard et al. 2016).

We refer the reader to Bolmont \& Mathis (2016) who investigated the impact of using this more realistic tidal dissipation model (in regards of a constant modified tidal quality factor) on the tidal evolution of close-in planets. This effect is also highly discussed in the proceeding contribution of Emeline Bolmont and collaborators for this conference. 


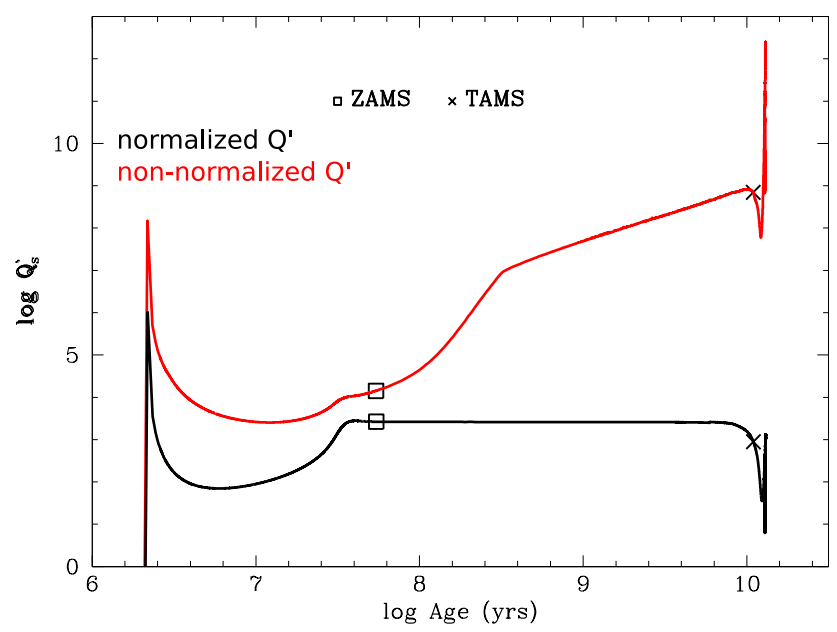

Fig. 6. Log of the modified quality factor as a function of time in the case of a fast rotating $1 M_{\odot}$ star $\left(P_{\text {init }}=1.4\right.$ days $)$. The black line represents the evolution of the modified quality factor at fixed normalized angular velocity (see Eq. (3) in Mathis 2015b) and the red line represent the non-normalised modified quality factor (see Eq. (1) in Mathis 2015b).

\section{Conclusions}

In this study we showed that the variations of the HZLs are important as stars evolve, and that they depend on stellar mass and metallicity that both strongly affect the stellar luminosity and effective temperature. We also showed that the HZLs are marginally affected by a change in rotation rate. However, while the stellar rotation does not impact the HZLs, it has a major role on the evolution of tidal and magnetic torques evolution (Mathis 2015b; Strugarek et al. 2015; Bolmont \& Mathis 2016) that then dramatically affects the orbital evolution of a given planet.

We have shown in Gallet et al. (2017) that a correlation exists between the magnetic stellar activity and location of the $\mathrm{HZ}$ along the stellar evolution. Indeed, the HZLs are the closest from the star when this latter is in a high active regime. During the MS phase, the minimum magnetic field required by an exoplanet for an efficient magnetospheric protection will increase for decreasing stellar mass. This would pose a problem in finding habitable planets around M-dwarf stars that will thus need strong magnetic field to host any form of life. However, while the pressure balance calculation done in Vidotto et al. (2013) is the first step in estimating the planetary magnetic field, it is somehow too simplified which have the consequence of reducing the extent of our results. 
F.G., C.C., and L.A. acknowledge financial support from the Swiss National Science Foundation (FNS) and from the French Programme National National de Physique Stellaire PNPS of CNRS/INSU. F.G., C.C., and L.A. acknowledge financial support from the SEFRI project C.140049 under COST Action TD 1308 Origins and from the French Programme National National de Physique Stellaire PNPS of CNRS/INSU. E. B. acknowledges that this work is part of the F.R.S.-FNRS "ExtraOrDynHa" research project. S.M. and E.B. acknowledge funding by the European Research Council through ERC grant SPIRE 647383. This work was also supported by the ANR Blanc TOUPIES SIMI5-6 020 01, the Programme National de Planétologie (CNRS/INSU) and PLATO CNES grant at Service d'Astrophysique (CEA-Saclay).

\section{References}

Amard, L., Palacios, A., Charbonnel, C., Gallet, F., \& Bouvier, J., 2016, A\&A, 587, A105

Bolmont, E. \& Mathis, S., 2016, Celest. Mech. Dyn. Astron., 126, 275

Borucki, W.J., Koch, D., Basri, G., et al., 2010, Science, 327, 977

Broeg, C., Fortier, A., Ehrenreich, D., et al., 2013, in European Physical Journal Web of Conferences, Vol. 47, European Physical Journal Web of Conferences, 03005

Cranmer, S.R., \& Saar, S.H., 2011, ApJ, 741, 54

Decressin, T., Mathis, S., Palacios, A., et al., 2009, A\&A, 495, 271

Donati, J.-F., Forveille, T., Collier Cameron, A., et al., 2006, Science, 311, 633

Folsom, C.P., Petit, P., Bouvier, J., et al., 2016, MNRAS, 457, 580

Gallet, F., \& Bouvier, J., 2013, A\&A, 556, A36

Gallet, F., \& Bouvier, J., 2015, A\&A, 577, A98

Gallet, F., Charbonnel, C., \& Amard, L., 2016, Origins of Life and Evolution of the Biosphere, 46, 395

Gallet, F., Charbonnel, C., Amard, L., et al., 2017, A\&A, 597, A14

Howell, S.B., Sobeck, C., Haas, M., et al., 2014, PASP, 126, 398

Kasting, J.F., Toon, O.B., \& Pollack, J.B., 1988, Scientific American, 258, 90

Kasting, J.F., Whitmire, D.P., \& Reynolds, R.T., 1993, Icarus, 101, 108

Kopparapu, R.K., Ramirez, R.M., SchottelKotte, J., et al., 2014, ApJ, 787, L29

Maeder, A., \& Zahn, J.-P., 1998, A\&A, 334, 1000

Mathis, S., 2015a, in SF2A-2015: Proceedings of the Annual meeting of the French Society of Astronomy and Astrophysics, ed. F. Martins, S. Boissier, V. Buat, L. Cambrésy, \& P. Petit, 401

Mathis, S., 2015b, A\&A, 580, L3

Mathis, S., \& Zahn, J.-P., 2004, A\&A, 425, 229

Matt, S.P., Brun, A.S., Baraffe, I., Bouvier, J., \& Chabrier, G., 2015, ApJ, 799, L23

Ogilvie, G.I., 2013, MNRAS, 429, 613

Palacios, A., Charbonnel, C., Talon, S., \& Siess, L., 2006, A\&A, 453, 261

Palacios, A., Talon, S., Charbonnel, C., \& Forestini, M., 2003, A\&A, 399, 603

Rasool, S.I., \& de Bergh, C., 1970, Nature, 226, 1037

Rauer, H., Catala, C., Aerts, C., et al., 2014, Exper. Astron., 38, 249 
Ricker, G. R., Winn, J. N., Vanderspek, R., et al., 2015, J. Astronom. Telesc., Instrum. Syst., 1, 014003

Russell, C.T. 2003, Planet. Space Sci., 51, 731

Strugarek, A., Brun, A.S., Matt, S.P., \& Réville, V., 2014, ApJ, 795, 86

Strugarek, A., Brun, A.S., Matt, S.P., \& Réville, V., 2015, ApJ, 815, 111

Truitt, A., Young, P.A., Spacek, A., Probst, L., \& Dietrich, J., 2015, ApJ, 804, 145

Valle, G., Dell'Omodarme, M., Prada Moroni, P.G., \& Degl'Innocenti, S., 2014, A\&A, 567, A133

Vidotto, A., Fares, R., Jardine, M., Moutou, C., \& Donati, J.-F., 2015, IAU General Assembly, 22, 2232894

Vidotto, A.A., Jardine, M., Morin, J., et al., 2013, A\&A, 557, A67

Zahn, J.-P., 1992, A\&A, 265, 115 